\title{
Editorial
}

Dermatology

\section{'Bullous' Pemphigoid: What Are You? Urgent Need of Definitions and Diagnostic Criteria}

\author{
Dan Lipsker ${ }^{\mathrm{a}}$ Luca Borradori ${ }^{\mathrm{b}}$ \\ ${ }^{a}$ Faculté de Médecine et Clinique Dermatologique, Hôpitaux Universitaires de Strasbourg, Strasbourg, France; \\ ${ }^{b}$ Department of Dermatology, University Medical Hospital Insel, Berne, Switzerland
}

The name 'bullous pemphigoid' (BP) itself is a pleonasm as 'pemphigoid' is derived from Greek and means 'form of a blister' (pemphix, blister, and eidos, form). Hence, from a purely etymological point of view, the adjective 'bullous' should not be added to designate the blistering in pemphigoid. Now, when can we make the diagnosis of 'classic' BP in our practice? Definitely, in all patients with a generalized pruriginous bullous eruption affecting predominantly the inner parts of the limbs and the trunk, in whom distinctive histopathological (that is subepidermal blistering, eosinophil infiltration of the upper dermis and/or eosinophilic spongiosis) and immunopathological findings (both positive direct immunofluorescence, DIF, studies and detection of circulating autoantibodies directed against the target antigens of BP, BP180 and BP230) can be demonstrated. However, what about the multiple 'non-bullous' variants of BP described under a variety of confusing terms $[1,2]$ like vesicular, prurigo-like and nodular, dyshidrotic, non-bullous BP, eczematous or erosive, erythrodermic, vegetans...? These 'atypical' variants, which most likely represent multiple facets of the same disease, require a high degree of suspicion to be diagnosed based on typical immunopathological features [1,2]. More importantly, at least in our experience, it is even conceivable that these 'atypical' variants together represent the most frequent forms of presenta-

\section{KARGER}

Fax +4161306 1234

E-Mail karger@karger.ch

www.karger.com
(C) 2010 S. Karger AG, Basel

$1018-8665 / 10 / 2212-0131 \$ 26.00 / 0$

Accessible online at:

www.karger.com/drm tions of BP compared to classic 'bullous' pemphigoid. In this issue of Dermatology, Geiss Steiner et al. [3] report 3 elderly female patients with a long history of widespread fibrinonecrotic ulcerations on the trunk and the limbs. Unexpectedly, some immunopathological findings suggested a diagnosis of BP. The problem arising is now to know whether either isolated DIF studies or detection of circulating autoantibodies are sufficient on their own to classify these patients as having BP or not.

Most entities in dermatology are usually diagnosed on the basis of their clinicopathological features, that is diagnosis relies on a typical clinical picture in combination with a corresponding characteristic or, at least, compatible histopathological image. In the area of autoimmune bullous diseases (AIBDs) however, DIF microscopy studies together with indirect immunofluorescence (IIF) studies and/or ELISAs are considered to be the 'gold standard' for diagnosis and classification. In this context, two questions have to be addressed: first, what are the diagnostic criteria of BP, and, second, how reliable is the diagnosis of BP in case of atypical clinical presentations, in which immunopathological findings are incomplete? First of all, besides a consensus statement about both definitions of disease activity and therapy response in $\mathrm{BP}$, we also lack well-accepted diagnostic criteria for BP. In elderly patients with the classic generalized bullous erup- 


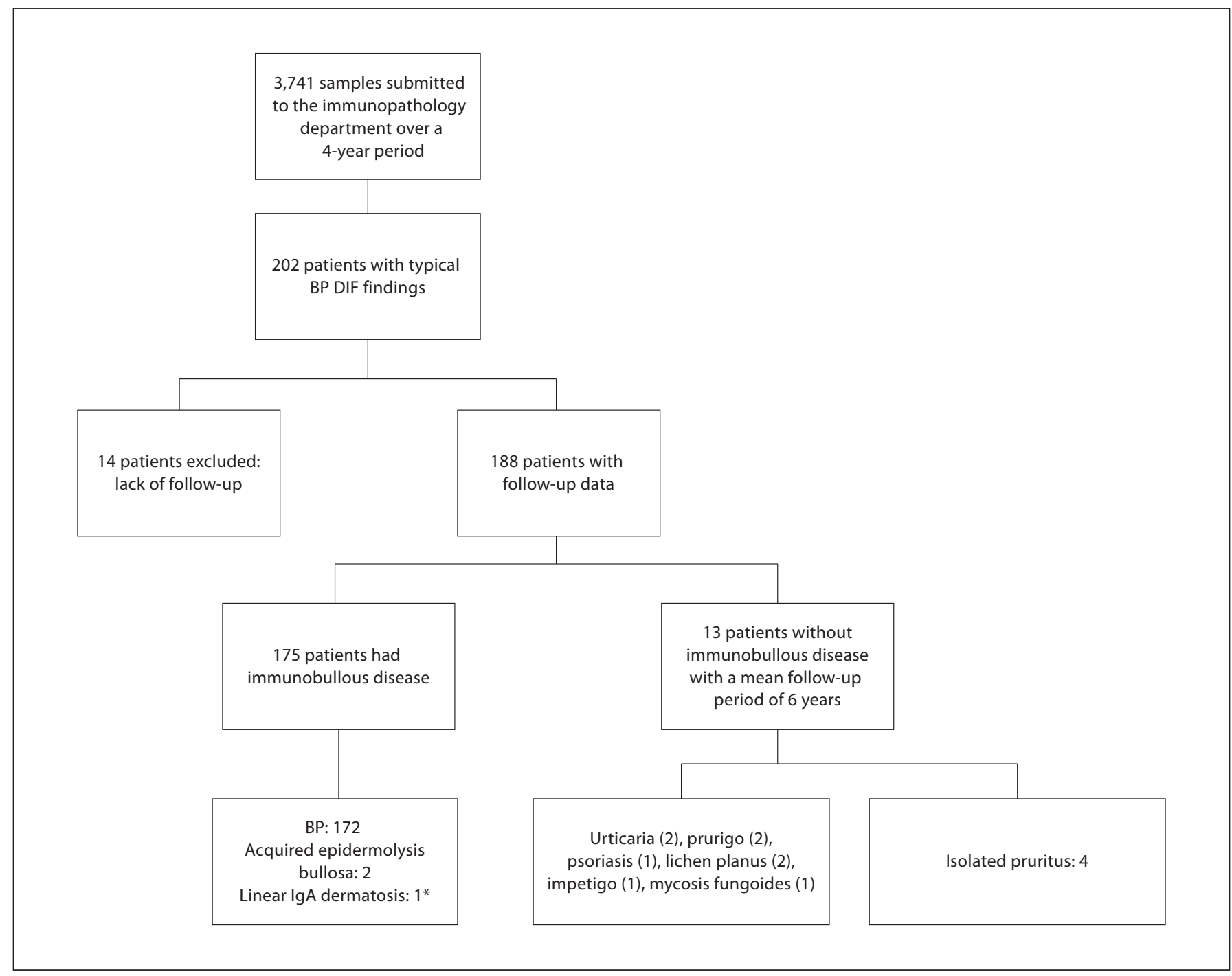

Fig. 1. Final diagnosis in 188 patients with a typical DIF finding of BP (fine and linear fluorescence along the dermal-epidermal junction with anti-IgG and/or anti-C3 antibodies) evaluated in Strasbourg, France. ${ }^{*}$ The patient with linear IgA dermatosis had also weak IgG deposition along the basement membrane.

tion, diagnosis is usually straightforward. In these patients, Vaillant et al. [4] and Joly et al. [5] have shown that clinical criteria are also very useful for a proper diagnosis. Indeed, when 3 of 4 distinct characteristics (absence of atrophic scars, absence of head and neck involvement, absence of mucosal involvement and age greater than 70 years) are present in a patient with linear deposits of IgG and/or C3 along the epidermal basement membrane by DIF microscopy, the diagnosis of BP can be established with a sensitivity of $90 \%$, a specificity of $83 \%$ and a high positive predictive value. In this context only, DIF microscopy is sufficient for a diagnosis of BP. However, what is the value of the detection of either linear deposits of immunoreactants along the basement membrane zone or of circulating autoantibodies in patients with eczematous, urticarial, excoriated or ulcerated lesions without obvious blistering such as in the patients described by Geiss Steiner et al.? The clinical diagnostic criteria for BP provided by Vaillant et al. [4] and Joly et al. [5] may be applied to patients with positive DIF studies, although in a small percentage of cases $(<10 \%$ ?) the detection of linear deposits of immunoreactants does not imply a diagnosis of BP, but of other AIBDs, such as epidermolysis bullosa acquisita anti-p200 pemphigoid. The diagnosis of BP can 
be of course supported by more detailed, time-consuming and/or more expensive analyses such as DIF studies performed on split skin, analysis of the specific staining pattern ( $\mathrm{N}$ - versus $\mathrm{U}$-serrated pattern), by performing fluorescence overlay microscopy studies, immunoelectron microscopy studies and/or the characterization of the specificity of circulating autoantibodies $[1,2]$. These studies would, more or less easily, allow to distinguish BP from other AIBD entities.

We have recently reviewed all DIF studies with fine and linear IgG and/or C3 deposits along the dermo-epidermal junction submitted to our immunopathology department in Strasbourg over a 4-year period. Such findings were found in 188 out of 3,471 patients' samples submitted for analysis. We obtained clinical and laboratory data from these patients, which are summarized in figure 1. Overall, the positive predictive value to diagnose an AIBD in the presence of this typical DIF pattern was $93 \%$. However, 13 of the 188 patients did not have any evidence of and did not develop an AIBD after a mean follow-up period of 6 years. Although this DIF pattern is highly suggestive of an AIBD (not necessarily BP), it is not an absolute proof. Hence, both clinical findings and overall context should be consistent with an AIBD for a definite diagnosis. The same reasoning now also applies to the detection of circulating antibodies. Since autoantibodies binding to proteins of the epidermal basement membrane are detectable in healthy individuals as well as in patients with a variety of pruritic skin disorders, whatever the method of screening (e.g. IIF studies, ELISAs or Western blot) [1, 2, 6-9], the presence of a seroreactivity should always be interpreted according to the clinical context and the results of DIF studies (the only exception is gestational pemphigoid, in which strong positive BP180 ELISA values may replace DIF studies [10]). In patients with atypical clinical findings, such as those reported by Geiss Steiner et al. [3], in our view the presence of circulating autoantibodies by either ELISA or IIF studies does not allow to make a diagnosis of BP if DIF microscopy studies are negative. Nevertheless, these cases have at least the merit to remind us of the importance to consider a non-bullous, 'atypical' variant of an AIBD in elderly patients with chronic itchy skin disorders, even in those in whom almost nothing else suggests this diagnosis a priori. In the absence of accepted criteria, it is up to the clinician, and his 'nosological sensitivity', to decide if an elderly patient with itch or prurigo, without bullae but with a positive DIF finding, suffers or not from BP or another AIBD. Finally, it is yet unclear which factors determine these 'atypical' modified presentations of BP (and

other AIBDs). A number of individual variables, such as age, co-morbidities, immunological and inflammatory response genes and environmental factors (e.g. drug, toxin, infection) are likely to have a critical impact on the final clinical phenotype of an AIBD. The situation is probably similar to what can be observed in patients with inherited epidermolysis bullosa carrying the same genetic mutations but showing different phenotype severities [11].

Finally, can we still talk about 'bullous' pemphigoid in a patient without bullae? The naming of a disease involves phenotypic description and knowledge about its natural history, i.e. prognosis. We do have these pieces of information in the case of typical BP, but not in that of the atypical variants. Furthermore, the characteristic elementary lesions of BP are erythema and bullae. Therefore, until a working set of diagnostic criteria for a definite and a probable diagnosis of $\mathrm{BP}$ is proposed, should we not require the presence of at least 1 bulla to fulfil the pleonastic designation of 'bullous pemphigoid'? The current efforts of an international group of experts and enthusiastic researchers in the field will hopefully provide mutually acceptable common definitions for pemphigoid to facilitate future epidemiological studies and clinical trials with the ultimate aim to provide better patient care.

\section{Acknowledgements}

This work was supported by grants from the Swiss National Foundation for Research (3100-067860) to L.B. and from the Coordination Theme 1 (Health) of the European Community's FP7, grant agreement No. HEALTH-F2-2008-200515 to L.B.

References

Dermatology 2010;221:131-134
1 Laffitte E, Borradori L: Bullous permphigoid and related disorders; in Hertl M (ed): Autoimmune Diseases of the Skin. Wien, Springer, 2005, pp 71-93.

2 Borradori L, Bernard P: Pemphigoid group (bullous pemphigoid, cicatricial pemphigoid, epidermolysis bullosa acquisita); in Bolognia JL, Jorizzo JL, Rapini RP (eds): Dermatology. London, Elsevier Science, 2006, pp 463-477.

3 Geiss Steiner J, Trüeb RM, Kerl K, Mühleisen B, French LE, Hofbauer GFL: Ecthyma-gangrenosum-like bullous pemphigoid. Dermatology DOI: 10.1159/000316098.

4 Vaillant L, Bernard P, Joly P, et al: Evaluation of clinical criteria for the diagnosis of bullous pemphigoid. Arch Dermatol 1998;134: 1075-1080 
5 Joly P, Courville P, Lok C, Bernard P, Saiag P, Dreno B, Delaporte E, Bedane C, Picard C, Sassolas B, Plantin P, D'Incan M, Chosidow O, Pauwels C, Lambert D, Loche F, Prost C, Tancrede-Bohin E, Guillaume JC, Roujeau JC, Gilbert D, Tron F, Vaillant L, French Bullous Study Group: Clinical criteria for the diagnosis of bullous pemphigoid: a reevaluation according to immunoblot analysis of patient sera. Dermatology 2004;208:16-20.

6 Rieckhoff-Cantoni L, Bernard P, Didierjean L, Imhof K, Kinloch-de Loës S, Saurat JH: Frequency of bullous pemphigoid-like antibodies as detected by Western-blot analysis in pruritic analyses. Arch Dermatol 1992; 128:791-794.
7 Hachisuka H, Kurose K, Karashima T, Mori O, Maeyama Y: Serum from normal elderly individuals contains antibasement membrane zone antibodies. Arch Dermatol 1996; 132:1201-1205.

8 Desai N, Allen J, Ali L, Venning V, Wojnarowska F: Autoantibodies to basement membrane proteins BP180 and BP230 are commonly detected in normal subjects by immunoblotting. Australasian J Dermatol 2008;43:137-141.

9 Wieland CN, Comfere NI, Gibson LE, Weaver AL, Krause PK, Murray JA: Anti-bullous pemphigoid 180 and 230 antibodies in a sample of unaffected subjects. Arch Dermatol 2010;146:21-25.
10 Powell AM, Sakuma-Oyama Y, Oyama N, Albert S, Bhogal B, Kaneko F, Nishikawa T, Black MM: Usefulness of BP180 NC16a enzyme-linked immunosorbent assay in the serodiagnosis of pemphigoid gestationis and in differentiating between pemphigoid gestationis and pruritic urticarial papules and plaques of pregnancy. Arch Dermatol 2005; 141:705-710.

11 Bodemer C, Tchen SI, Ghomrasseni S, Séguier S, Gaultier F, Fraitag S, de Prost Y, Godeau G: Skin expression of metalloproteinases and tissue inhibitor of metalloproteinases in sibling patients with recessive dystrophic epidermolysis and intrafamilial phenotypic variation. J Invest Dermatol 2003;121:273-279. 
